\title{
La organización de la Hacienda Real en la provincia de Chiapa (1540-1644): OFICIALES REALES, SUBDELEGADOS Y DEMÁS PERSONAJES
}

\author{
The organization of the Hacienda Real in the Province of Chiapa (1540-1644): \\ royal officials, delegates and other personages
}

Ana María Parrilla-Albuerne

Resumen: El objetivo principal de este trabajo es analizar el desarrollo de la organización hacendística de la provincia de Chiapa durante los siglos XVI y XVII para de esta forma entender el desarrollo de la administración local y cómo se fueron tejiendo las relaciones entre los diferentes grupos de poder. El aparente aislamiento y la falta de atractivos económicos permitieron a los oficiales reales de la Audiencia de Guatemala nombrar a un subdelegado con el título de teniente de oficiales reales que, según nuestra opinión, es una pieza clave para comprender el desarrollo de la administración provincial y cómo se fueron entrelazando las relaciones entre los diferentes grupos del poder local.

Palabras clave: Real Hacienda, subdelegados, elites, familias, tributos.

Abstract: The main objective of this work is to analyze the development of the treasury organization in the province of Chiapa during the $X V I$ and XVII centuries, in order to understand the development of the local administration and how the relationships between the different groups of power were weaving. The apparent isolation and lack of economic attractions, allowed to the royal officers of the Audience of Guatemala, to appoint a minor delegate with the title of teniente de oficiales reales which, in our view, is a key piece to understand the development of the provincial administration and how the relationships between the different groups of local power were intertwining.

Keywords: Real Hacienda, minor delegates, elites, families, tributes.

Ana María Parrilla Albuerne, doctora por la Universidad Complutense de Madrid. Profesora-investigadora de la Universidad de Ciencias y Artes de Chiapas. Temas de especialización: relaciones de poder y organización hacendística en el Chiapas colonial, arte colonial. Correo electrónico: albuerne_ana@yahoo.com.mx.
Enviado a dictamen: 05 de marzo de 2013.

Aprobación: 20 de abril de 2013.

Revisiones: 1. 
$\mathrm{D}$ urante los siglos XVI y XVII la organización de la hacienda en Indias prácticamente no experimentó cambios aunque, según indica Águeda Jiménez Pelayo (2001: 133), durante el reinado de Felipe II (1556-1598) hubo algunas modificaciones. Poco a poco el sistema administrativo alcanzó cierta estabilidad, como señala Horst Pietschmann (1996: 115); sin embargo, aunque se tomaron ciertas medidas para adaptar el aparato gubernamental a las nuevas situaciones, éstas fueron insuficientes.

Luis Jáuregui (1999: 24) indica que, durante los siglos XVI y XVII, desde el punto de vista financiero se vivieron dos fenómenos contrapuestos: por un lado, un intento de centralización mediante la creación de órganos administrativos propios - es el caso de los "secretarios" de Estado y del Despacho-, lo cual hizo más grande la burocracia fiscal americana en relación con la española. $\mathrm{Y}$, por otro, un fenómeno de descentralización que se caracterizó por la creciente venta de cargos con funciones recaudadoras y contadoras. La venalidad de la Corona, en vista de las crecientes necesidades financieras, trajo como consecuencia la colusión entre autoridades recaudadoras y oligarquías locales, así como una mayor corrupción y, consecuentemente, ineficiencia. Esto acompañado de cambios económicos que produjeron una mayor especialización de las actividades económicas a nivel regional.

Según François Xavier Guerra (1998: 120), la vida social y política de estos siglos se caracteriza porque el grupo tiene prioridad sobre el individuo, el hombre forma parte de un todo con vínculos permanentes. Las acciones individuales remiten a un grupo, por lo que la sociedad tradicional se caracterizó por la fuerza de las corporaciones. Entre estas últimas, debemos considerar al cabildo, ocupado durante la primera parte de la Colonia por las oligarquías locales, y a los oficiales reales, los cuales han sido objeto de estudio por parte de Michel Bertrand (2011).

Teniendo en cuenta estos antecedentes, en este trabajo se realizará un primer acercamiento al estudio de la organización hacendística en la provincia de Chiapa durante los siglos XVI y XVII, tema que aún no ha sido abordado en profundidad. Además, pretendemos hacer un acercamiento preliminar a la forma en que esta estructura fue utilizada por los diferentes actores hasta desencadenar, entre 1680 y 1721, graves conflictos entre los distintos grupos de españoles; periodo, este último, analizado en profundidad por Viqueira (1994: 237-267).

\section{La administración fiscal en la provincia de Chiapa: 1540-1595}

Desde su conquista, la adscripción política de Chiapa fue un asunto delicado. Según Peter Gerhard (1991: 12), hasta 1529 tanto Chiapas como Tabasco estuvieron dentro de la jurisdicción de Guazacualco -Espíritu Santo-, en Nueva España. A principios de ese mismo año se reunió en la Ciudad de Mexico la Primera Audiencia, que envió a la provincia de Chiapa como alcalde mayor a don Juan Enríquez de Guzmán (Lenkersdorf, 2001: 214). Guzmán contó con autoridad para reasignar las encomiendas y los cargos de regidor y pacificar a los indios rebeldes. A mediados de 1531, Guzmán terminó su servicio en la provincia a consecuencia de que la Corona sostuvo que Chiapas formaba parte de la jurisdicción de Guatemala; de esta forma, la demarcación pasó a formar parte de la Capitanía General de Guatemala y desde ese momento los colonos ya no tuvieron la necesidad de recurrir a México con sus solicitudes (Lenkersdorf, 200la: 247). En 1540, la provincia de Chiapa fue obtenida por el adelantado Francisco de Montejo al intercambiarla por Honduras con Alvarado. El gusto le duró poco a Montejo, puesto que al constituirse la Audiencia de los Confines, en 1544, Chiapa fue incluida en su jurisdicción. Entre 1564 y 1569 la sede de la Audiencia se traslada a Panamá. Supuestamente, durante este lapso las apelaciones de Chiapa debían trasladarse a la Audiencia de México, pero el gobernador de Guatemala la siguió controlando. En 1569, cuando se establece la Audiencia de Santiago de Guatemala, Chiapa queda subordinada a ésta (Gerhard, 1991: 12).

La alcaldía mayor de Chiapa, creada en 1577, fue un territorio marginal que aportaba pocas riquezas a la Corona española. Este aislamiento se debía principalmente a los escasos atractivos que ofrecía a 
los colonizadores que llegaban a este territorio, según Viqueira (2000: 105).

Para hablar de una fiscalidad debemos reconocer la existencia de una autoridad fiscal que cumpla tres funciones en este sistema: que organice, gestione y cobre (Artola, 1982: 21). Como sabemos, en el caso de América la Casa de la Contratación fue la primera encargada de organizar y recaudar los fondos de la Real Hacienda como representantes del rey; sin embargo, la existencia de autoridades fiscales intermedias limitaba este esquema. Las preguntas que aquí planteamos son: ¿quiénes cobraron y administraron los impuestos de la Real Hacienda en Chiapa? y ¿cómo se llevó a cabo esta labor?

Los fondos de la Real Hacienda se clasificaban en diversos ramos: impuestos sobre los actos tráfico y operaciones comerciales, producción minera, producción agrícola, concesión de empleos y beneficios-, impuestos sobre las personas, impuestos sobre las rentas estancadas e ingresos extraordinarios —venta de oficios, situados, servicios-. Los primeros gravámenes que se cobraron fueron el quinto real, el diezmo, el almojarifazgo, la alcabala y el tributo. Los ramos estancados, las bulas de la Santa Cruzada, los donativos y servicios —exigidos a particulares mediante empréstitos que muy pocas veces se restituían-, la venta de cargos públicos y la media anata, entre otros, complementaban los ingresos de la Corona (Ots, 1945: 384-387).

Entre los impuestos sobre los actos cabría destacar el cobro del quinto real. Este gravamen se estableció en febrero de 1504, consistía en el pago de la quinta parte de la toma o extracción de metales en las Indias y se mantuvo hasta el siglo XVIII. En el caso de la provincia de Chiapa, y a pesar de las carencias anteriormente mencionadas, existen algunas evidencias que nos hablan de la existencia de una mina en el hoy abandonado pueblo de Copanaguastla (Ruz, 1992a; Viqueira, 2002: 122), sin que se haya podido determinar su localización exacta. La producción de dicha mina, sin descartar la existencia de alguna otra, no debía ser nada despreciable, puesto que en dos reales cédulas de $1535^{1}$ y $1536^{2}$ se ordena a los oficiales reales de Guatemala que acudan y estén presentes en la fundición de la villa de San Cristóbal de los Llanos, nombre de lo que hoy es San Cristóbal de Las Casas. Como señala Mario Humberto Ruz, en 1539 se notifica al virrey, don Antonio de Mendoza, que se había aprobado la solicitud de los vecinos de Ciudad Real para que en ella residiera un platero que pudiera vigilar la fundición de oro. Además, señala que existen pocos datos que nos indiquen el desarrollo y localización de la actividad minera en la zona (1992a: 75).

Hasta el momento poco se sabe las cantidades exactas de metal obtenidas. La única cita que existe al respecto es la que hace Robert S. Chamberlain (1948: 178), quien habla de 38500 pesos enviados a Guatemala en el año 1541. Esta cantidad, para 1549, había descendido a 10170 pesos, ${ }^{3}$ que sumaba todo lo recaudado en los diferentes ramos durante aquel año. Según Mario Humberto Ruz, "la producción, al menos durante unos años, debió parecer importante puesto que Montejo construyó en Ciudad Real una casa de fundición, como lo indica en su propia probanza" (Ruz, 1992a: 76; Chamberlain, 1948: 199, nota 83). Lo que parece claro es que estas actividades hicieron necesaria la creación de una caja de la Real Hacienda en Chiapa, de la que existen documentos fechados entre los años 1540 y 1549. En estos documentos se especifica que los oficiales reales encargados de ella eran Diego Vázquez de Ribadeneira, contador, García de Mendaño, tesorero, y Antonio de la Torre, factor y veedor. ${ }^{4}$

Los oficiales reales eran los responsables de recaudar los impuestos, de distribuir los ingresos y de pagar a los proveedores, y contaban con ciertas características que los convertían en pieza fundamental en el funcionamiento de la administración novohispana. En el caso de Chiapa, vemos que estos primeros oficiales, según las cuentas que dieron en 1550 a Cerrato presidente de la Audiencia de los Confines-, se encargaban de recaudar principalmente "los tributos de los pueblos de su Majestad como de las fundiciones e penas de cámara e diezmos e de la plata." ${ }^{5}$

De todos los rubros apuntados, en el documento señalado sólo aparece información de la fundición de oro y plata y del cobro de depósito de las penas de los 
casados $^{6}$ "que tienen sus mujeres en Castilla", como se observa en la tabla 1 .

Desde 1550 no hemos encontrado ningún tipo de cuentas dadas por la Caja Real de Chiapa a la Corona. Esto nos hace pensar que quizá ésta desapareció y la administración de la hacienda en esta provincia pasó a depender de la Real Caja de Guatemala, donde los oficiales reales se hicieron cargo del cobro y de la administración de impuestos. Recordemos que las cajas reales eran fundadas básicamente en los lugares donde existían minas o puertos de mar, de manera que cuando la veta de oro pareció agotarse, fue clausurada ésta.

Una vez que la actividad minera decreció en la región, se hizo más evidente que "la actividad económica tenía que depender necesariamente de la cantidad de población explotable" (Viqueira, 1994: 241).

Según la legislación colonial, las tierras, las aguas, los montes y los pastos eran regalías de la Corona que los particulares podían usufructuar a través de las mercedes. De esta forma, durante la conquista, mas no durante la colonización, el repartimiento fue esencial para que los castellanos obtuvieran los títulos de propiedad de determinados bienes; recordemos que hasta 1532 no se declaró que los encomenderos no tenían dominio directo sobre los indios - ya que esto le correspondía al rey-.

De esta manera en real cédula de 17 de agosto de 1529 , se fundaba la libertad de los indios, prohibió a los encomenderos que alquilaran o dieran prestados lo que les correspondían. La Audiencia, con autorización de la Corona, puso en práctica la tasación de las encomiendas, o sea, la determinación de lo que los indios debían contribuir a sus respectivos encomenderos (Zavala, 1992: 63).

Los jefes de las expediciones tenían la facultad de repartirlas, y los beneficiados debían cultivarlas y residir en el distrito. Posteriormente, a lo largo del siglo XVI, la encomienda se convirtió en una merced otorgada como recompensa a conquistadores y pobladores. Los encomenderos percibían los tributos indígenas de las poblaciones comprendidas en su título y tenían que residir en la jurisdicción y destinar éstos a mantener caballos y armas para la defensa del territorio y al adoctrinamiento y la enseñanza de los aborígenes. Un caso de pueblo tributario directamente de la Corona en la provincia de Chiapa es Chiapa de Indios, que a partir de 1552 pasó a denominarse Chiapa de la Real Corona. Desgraciadamente, no contamos con un estudio profundo de las encomiendas de Chiapa durante los siglos XVI y XVII y esto no nos permite saber qué pueblos de Chiapa eran tributarios directos a la Corona; sin embargo, en el documento sobre las cuentas que los oficiales reales de la caja real de Chiapa dieron a Cerrato en 1550, todo parece indicar que los pueblos de la provincia estaban incluidos en el sistema de encomiendas y, por lo tanto, el cobro de los tributos de los pueblos se efectuaba por los encomenderos.

Tadashi Obara-Saeki (2010) realizó un estudio muy interesante sobre demografía tributaria partiendo del análisis de diferentes etapas en la tasación y cobro de tributos en Chiapas; sin embargo, este trabajo fue revisado, en parte, en un artículo posterior en el cual el autor propone una nueva periodización basada en los criterios para la definición de tributarios y el modo en que se calculaba su número (Obara-Saeki, 2012: 38 40). De ambos escritos se pueden extraer diferentes periodos dependiendo del aspecto de la tributación que queramos resaltar. Nosotros elegimos la realizada por el autor en su primera publicación, de 2010, porque consideramos que estas etapas son un intento de concretar los cambios de acuerdo con la tasación y, lo más importante para nosotros, el cobro de tributos en la entidad.

De esta forma, el autor indica que existieron varias etapas: una primera, que fecha entre 1524 y 1541, en la cual la labor de cobro de tributos estaría en manos de encomenderos sin que existiera una tasación oficial del tributo (Obara-Saeki, 2010: 76-92).

Durantelos primeros 15 años, no existió prácticamente ninguna reglamentación que rigiera el monto y el modo de la tributación, así que cada encomendero podría determinar, de acuerdo con los caciques y principales, las condiciones de la tributación — qué 
especies, cuánto y cada cuánto tiempo, etcétera(Obara-Saeki, 2012: 38).

El autor pone como ejemplo en este punto a Baltasar Guerra, encomendero del pueblo de Chiapa, cuyos abusos hicieron que se desencadenaran fuertes descontentos entre la población; sin embargo, es importante aclarar que Baltasar Guerra llegó en 1532 a la provincia como segundo teniente de gobernador nombrado por Pedro de Alvarado ${ }^{7}$ (Lenkersdorf, 200la: 250) y que fue precisamente el primer tesorero de la Caja Real de Chiapa:

[...] cuentas de la dicha real caja de hacienda que a su majestad perteneció en la provincia de Chiapa después de que en ella hubo oficiales de su majestad porque desde antes no se halló razón alguna más que de los oficiales de Guatemala usaban allí por subtenientes los dichos oficios hasta tanto que el Visorrey de la Nueva España don Antonio de Mendoza por especial comisión [ilegible] su majestad para ello tuvo proveído de tesorero a Baltasar Guerra e de contador a Diego Vázquez Ribadeneyra e de factor Antonio de la Torre e después por ausencia del dicho Baltasar Guerra usó el dicho oficio de tesorero García de Mendaño vecino de aquella provincia. ${ }^{8}$

Los tesoreros eran la máxima autoridad entre los oficiales reales de las cajas, depositarios del libro de cuentas que se dividía en el número de ramos que se administraban en la caja. En estos libros se debía asentar cada movimiento concerniente al ramo en cuestión para posteriormente ser actualizados; sin embargo, como indica Michel Bertrand, las posibilidades de fraude eran numerosas (Bertrand, 2011: 89). En efecto, ni Baltasar Guerra ni posteriormente Diego Vázquez de Rivadeneyra rindieron cuentas de sus ingresos por tributos, el ramo que en el caso de Chiapa era el que más beneficios proporcionaba.

Desde el punto de vista de la tasación y el cobro de tributos en Chiapa, Obara-Saeki refiere una nueva etapa, entre 1541 y $1571,{ }^{9}$ la cual se inaugura con la primera tasación de tributarios en Chiapa realizada en 1541 por el obispo Francisco Marroquín, otras tantas se llevaron a cabo entre 1560 y 1562 asignando tributos excesivos (2010: 86). Es precisamente en estas últimas tasaciones cuando se observa un cambio importante en la forma de contar los tributarios o, mejor dicho, un primer intento para identificar quién era un tributario:

Se consideraba tributario a un varón casado que habitualmente poseía casa, solar y milpa, lo que le permitía mantener una familia y pagar tributo... es probable que quienes tenían casa [aunque fuesen viudos] y milpa se hayan contado como tributarios (Obara-Saeki, 2012: 39).

En el caso concreto de Baltasar Guerra, el tesorero de la Caja Real de Chiapa entre 1541 y 1545, es posible que los indios de Chiapa obedecieran a Guerra no sólo por "el miedo que fue impuesto después de las dos 'guerras' que tuvieron con él y de la 'justicia' que tuvieron después" (Obara-Saeki, 2010: 86), sino también por el puesto que ocupaba dentro de la administración y que haría entendible el consejo que los dominicos dieron a los habitantes de Chiapa:

[...] solamente le debéis dar los tributos que están tasados, no porque en [sic] vuestro señor sino porque el rey lo manda así, por los servicios que le han hecho; y pagado aquello, no tenéis más que ver con él; y si más os pidiere hay justicia que lo castigue (ObaraSaeki, 2010: 87).

Consideramos que a partir de 1550, año en el que desaparece esta primera Caja Real de Chiapa, ${ }^{10}$ hasta 1580, cuando Juan de Mesa Altamirano - primer alcalde mayor de Chiapa - realiza su visita a todos los pueblos ${ }^{11}$ de la alcaldía mayor, el cobro y la administración de tributos de aquellos pueblos que no habían pasado a la Corona, siguieron estando en manos de los encomenderos. Es cierto que desde el punto de vista de la tasación de tributos podemos hablar del inicio de una tercera etapa a partir de 1570 o 1571 (ObaraSaeki, 2010: 92; 2012: 39); sin embargo, desde el punto de vista administrativo debería extenderse hasta 1580, 
cuando el cobro de tributos pasó a ser ejercido por los alcaldes mayores y corregidores, según nuestra opinión. Es importante señalar en este punto que existe un casi total desconocimiento del papel jugado por los corregidores en el cobro de tributos, aunque contamos con alguna información que puede servir para estudios posteriores más amplios y que retomaremos en el siguiente apartado.

Saeki-Obara propone que 1570 marca el inicio de una nueva etapa en la tasación de tributos que duraría, según su opinión, hasta 1616. Durante este periodo se comenzó a contar como

[...] medios tributarios a los viudos de ambos sexos y a los solteros que no vivieran bajo la potestad paterna. Durante las dos décadas posteriores parece que los hombres viudos y solteros fueron considerados como "medios tributarios" y las mujeres viudas y solteras como "tercios tributarios"; es decir, cada tres de estos últimos formaban un "tributario entero" (ObaraSaeki, 2012: 39).

Entre 1580 y 1595, el cobro y la administración de tributos fueron ejercidos por los alcaldes mayores: Juan de Mesa Altamirano (1578-1582), García de Padilla (1581-1585), Pedro Martínez (1586-1592), Martín Nañez (1593) y Pedro Martínez (1594-1595); ${ }^{12}$ aunque sabemos que había excepciones, específicamente en el pueblo de Chiapa y en el barrio del Cerrillo (Lenkersdorf, 2001b: 162 y 206) existían corregidores encargados del cobro de tributos, como veremos más adelante. Es precisamente en este último año cuando los oficiales reales de la Caja de Guatemala se hacen cargo del cobro de tributos en la provincia, mediante el nombramiento del primer teniente de oficiales reales del que tenemos noticia en Chiapa: Diego de Alegría. Con él iniciaría un nuevo periodo de administración hacendística en la provincia.

\section{El corregidor. Una figura escasamente estudiada}

El cargo de corregidor hay que entenderlo a partir del debate que se generó en torno a la legalidad o ilegalidad de las encomiendas; es por ello que, como apuntamos en párrafos anteriores, nos parece tan importante un estudio extenso sobre la evolución de esta institución en la Capitanía General de Guatemala y, por ende, en la provincia de Chiapa. Exponer aquí la legislación y pareceres que se generaron en torno a esta discusión desde poco antes de la implantación de la Segunda Audiencia, en 1529 aproximadamente, excedería con mucho los objetivos de este artículo. Sin embargo, podemos aclarar que a partir de la implantación de la Segunda Audiencia se generaron dos posturas en torno al tema: por un lado, los defensores de la encomienda y, por otro, los de una administración regalista (Zavala, 1992: 57).

En una instrucción secreta presentada a la Audiencia, dice:

Yo vos mando, que luego que llegáredes os informeis de los indios que han vacado [...] todas las encomiendas que los susodichos Presidentey Oidores ovieren hecho de los indios que han vacado, las deis por ningunas...y vos mandamos, que luego los quitéis a las personas a quien estuvieren encomendados, y los pongáis dichos indios en libertad, señalándoles tributos que os pareciere, que pueden y deben pagar buenamente, con los cuales acudan a nuestros oficiales; y a ellos les haced cargo de todo ello; y pondréis personas hábiles, que sean tenidos por de buena conciencia, para que tengan en justicia a los dichos indios [...] Y lo mismo haréis de todos los que han vacado y vacaren en cualquier manera; hasta tanto que, vista vuestra relación, Nos vos enviemos a mandar lo que a nuestro servicio, bien y población de la dicha tierra convenga. Las personas que así se pusieren en los tales pueblos, se llamen corregidores, para que aún por el nombre conozcan los indios que no son sus señores (Encinas citado en Zavala, 1992: 57).

Como señala el propio Zavala, el corregimiento tenía como fin limitar el sistema administrativo de los indios por medio de las encomiendas; sin embargo, el corregimiento no fue incompatible con la encomienda, "sino uno de los medios para ordenar la relación entre encomendero y los indios" (1992: 57). La idea, a fin de 
cuentas, era que los oidores fueran los encargados de ir extinguiendo las encomiendas y de fundar los corregimientos. El 22 de junio de 1532 dio su parecer sobre el tema el doctor Ceynos, oidor de la Audiencia de México. Según su opinión "sólo quedaban dos caminos: o conceder las encomiendas, o establecer un sistema regalista absoluto, cobrando el rey sus rentas de los indios y gobernándolos por medio de los corregidores" (Zavala, 1992: 62).

La figura del corregidor, legislativamente hablando, fue bastante controvertida pues su aceptación suponía el fin de la perpetuidad de las encomiendas y el control de los tributos por parte de la Corona. Por ello, se van a ir modelando sus atribuciones y discutiendo la pertinencia de su existencia. Por ejemplo, en 1537, el virrey Mendoza advertía en una carta, respecto al gobierno de los indios de la Corona, que los corregidores no eran aptos, no tenían ningún cuidado de los indios y les robaban, y que era mejor poner alcaldes mayores:

[...] me parece [...] se quitasen de todo punto los corregidores y se les diese por vía de acostamiento, con qué sustentarse, y que cada uno de los alcaldes mayores que se pusiese, estuviesen cuidando de recoger todos los tributos de la provincia en que estuviesen y dar cuenta dellos a los oficiales de V.M. (Zavala, 1992: 70).

En 1567, Juan de Matienzo, oidor de la Audiencia de Charcas (Perú), trató el problema teórico de la justificación de las encomiendas y, en su texto, hace alusión a los corregidores y sus características. El corregidor debía residir en los pueblos donde hubiere cantidad de propios, tendría atribuciones de justicia,

[...] el litigio entre indios debía fallarlo sin escritura; cuando interviniera algún español, podría formar un proceso sumario, sin más trámites que la demanda, respuesta, testigos y sentencia. En causas criminales en que la sentencia fuera de muerte o mutilación de miembro podía haber apelación al cabildo de la ciudad más próxima o para la audiencia" (Zavala, 1992:173).
El corregidor residente en el pueblo debía tener al menos cuatro pueblos bajo su jurisdicción, con un total de 2000 indios y gozaría de un salario de 1000 pesos anuales que se pagarían la mitad por la tasa del rey y la otra mitad por el producto del esquilmo del ganado (Zavala, 1992: 173). Como podemos observar, las atribuciones de los corregidores fueron aumentando por la necesidad de la Corona de limitar las acciones de los encomenderos.

En el caso concreto de Chiapa sabemos que a mediados del siglo XVI existían al menos dos corregimientos: el barrio del Cerrillo en Ciudad Real - hoy San Cristóbal de Las Casas- y el pueblo de Chiapa de la Real Corona - hoy Chiapa de Corzo(Lenkersdorf, 2001b: 162 y 206). Como se señala en la Recopilación de las Leyes de Indias de 1680, los corregidores eran los encargados del cobro de tributo ${ }^{13}$ y el envío de éste a los oficiales de la Real Hacienda. Es decir, el cobro de tributos en pueblos dependientes directamente de la Corona era realizado por los corregidores; sin embargo, como apuntamos en líneas anteriores, no tenemos conocimiento del número total de pueblos que tenían esta característica. Tampoco sabemos cuándo dejó de existir la figura del corregidor, pero tenemos al menos dos informaciones que nos dan algunas pistas preliminares: por un lado, sabemos que en el pueblo de Chiapa existía un corregidor en el año 1584, ya que aparece nombrado en la relación que hace el obispo fray Pedro Feria sobre la reincidencia en las idolatrías: ${ }^{14}$ y por otro, en 1623 se hace el inventario de bienes de Alonso Zapata de Cárdenas, quien desempeñó entre otros cargos el de corregidor del pueblo de Chiapa de los Indios. ${ }^{15}$

Ahora bien, una pregunta que se podría plantear es la siguiente: ipor qué la figura del corregidor siguió existiendo aún después del nombramiento de los alcaldes mayores? Según nuestra opinión, un tanto especulativa aún, la respuesta deberíamos encontrarla en la propia legislación. Precisamente, la dicotomía viene dada por el control de los tributos; los alcaldes mayores controlan, económica y jurídicamente, tanto los pueblos encomendados en los que los tributos habían sido otorgados por la Corona a particulares, como aquellos que no contaban con propios. Por otro 
lado, los corregidores dominan los pueblos o barrios que no se encontraban encomendados y, por lo tanto, eran tributarios directos de la Corona.

\section{El primer teniente de oficiales reales de la Caja Real de Guatemala en Chiapa: Diego de Alegría}

Hace tiempo inicié una investigación sobre cómo se había llevado a cabo el cobro de tributos en la provincia de Chiapa con base en el pleito que entabló don Manuel de Maisterra y Atocha, alcalde mayor de Chiapa desde 1685, contra los oficiales reales de la Audiencia de Guatemala (Parrilla, 2011: 13-32). Este documento nos ofrece una gran cantidad de datos para comprender el complejo sistema tejido por los oficiales reales para hacerse con la administración y el cobro de tributos en la provincia de Chiapa, lo cual consiguieron mediante el nombramiento de un teniente de oficiales reales. ${ }^{16}$

Las leyes de Indias eran explícitas en cuanto a la normatividad que regía el nombramiento de tenientes de oficiales reales; por ejemplo, no debía dejarse el cobro de tributos y rentas reales a los tenientes de oficiales reales más allá de cinco leguas a la redonda de donde existiera una Caja Real. Rebasada esta distancia, el cobro lo debían efectuar los alcaldes mayores. Existía también en estas leyes la prohibición de nombrar tenientes por los oficiales reales exceptuando por ausencia o enfermedad (Parrilla, 2011: 20). Sin embargo, el hecho de que Chiapa se encontrara aislada en todos los sentidos y que aparentemente no contara con atractivos económicos fue determinante para que durante largos periodos la Corona no le prestara la atención debida, pese a que el ingreso por cobro de tributos se volvió el ramo más importante. Esto permitió a los oficiales reales de la Real Caja de Guatemala nombrar a un subdelegado con el título de teniente de oficiales reales que, según nuestra opinión, es una pieza clave para comprender el desarrollo de la administración local y cómo se fueron tejiendo las relaciones entre los diferentes grupos del poder local.

Dada la complejidad del documento anteriormente mencionado, en el que además de las tensiones entre el alcalde mayor, Manuel de Maisterra y Atocha, y los oficiales reales, Miguel de la Vega Balbuena y Felipe de Maiz y Lizárraga, se presentan muchos otros datos sobre el manejo del aparato administrativo local en Chiapa, decidimos extender nuestra investigación hasta la aparición del primer teniente de oficiales reales del que se tiene noticia en la provincia de Chiapa, Diego de Alegría.

Como afirma Michel Bertrand para el caso de la Nueva España, la densidad de la malla administrativa de la Real Hacienda era insuficiente (Bertrand, 2011: 75). Es más, desde la Caja Real de la Ciudad de México y la de Santiago de Guatemala existía un enorme vacío administrativo. Esta dispersión geográfica, junto con la multiplicación de obligaciones, hizo que, en el caso de la Nueva España, muchas cajas reales buscaran estrategias para acrecentar la estructura existente creando segundos cargos de oficiales reales, como en el caso de El Parral -Caja Real de Durango-, con responsabilidades limitadas, enviando un representante o rotando a los oficiales reales en determinados destinos, como es el caso de Campeche - Caja Real de Mérida- (Bertrand, 2011: 76-77). Pareciera que estas mismas causas produjeron el nombramiento de Diego de Alegría como teniente de oficiales reales en la provincia de Chiapa, si no fuera porque éste no fue nombrado por los oficiales sino por el presidente de la Audiencia de Guatemala, Francisco de Sande, y el fiscal de su majestad, en 1595.

Lo que sabemos de Diego de Alegría y Mandojana es que era natural de Vitoria y que había llegado a Guatemala en 1594 como criado del recién nombrado presidente de la Audiencia de Guatemala. ${ }^{17}$ Un año después, en 1595, es nombrado juez y administrador de la Hacienda Real en la provincia de Chiapa, ${ }^{18}$ puesto que ocupó hasta 1597, cuando es reemplazado por Francisco del Padrón. En 1598 recupera sus funciones desempeñándolas ininterrumpidamente hasta 1644. Durante este periodo fue propuesto para varios oficios, como el de tesorero de Nicaragua o la Isla de Cuba y de Antioquía, en $1599,{ }^{19}$ y para ocupar la contaduría de Panamá en 1604:20 sin embargo, ninguno de ellos le fue otorgado. En 1629 se le concede el título de Caballero de la Orden de Santiago. ${ }^{21}$ En este documento se incluyen 
las pruebas presentadas y por ellas podemos conocer algunos datos de su familia, como que su padre fue durante muchos años notario de la ciudad de Vitoria y desempeñó diversos cargos en el ayuntamiento de la ciudad.

Entre las funciones que ejerció Diego de Alegría como teniente de oficiales reales se encuentran: el cobro de tributos reales y servicio del tostón ${ }^{22}$ y el cobro de alcabalas, precios de los oficios vendibles y remates de los dichos tributos. Éste no debía recibir sueldo, puesto que según la Recopilación de Leyes de Indias estaba usurpando las funciones del alcalde mayor, que era quien, según la ley, debía estar cobrando los tributos de la provincia. Los ingresos de esta provincia parecen haber sido más cuantiosos de lo que en un primer momento se pudo pensar:

[y en ninguna de] las provincias de nuestro distrito es más preciso y necesario teniente que en la de Chiapa porque allí concurren todas las formalidades de Caja Real que en ésta de Guatemala por la variedad de ramos de hacienda que se administran y por la mucha distancia que se pagan y satisfacen en aquella caja todos los establecimientos que en ésta lo cual no ocurre en otra alcaldía mayor ni corregimiento y por las leyes citadas concedemos que los nombramientos tocan a superiores del mismo efecto. ${ }^{23}$

Sin embargo, en 1689 se reconoce que el teniente de oficiales reales en Chiapa, Juan de Azcaray, habría cobrado estos tributos y servicios. (ver tabla 2).

En el año 1600 el presidente de la Audiencia de Guatemala, le encarga al teniente de oficiales reales la realización de un padrón de medidas y computaciónes de tierras, estancias y caballerías que contaran o no con títulos de asignación, tarea que llevó a cabo en algún momento entre 1598 y 1600, motivo por el cual Alegría volverá a pedir una retribución monetaria, aunque desconocemos si ésta le fue otorgada. En efecto, a lo largo del siglo XVI se dio en la provincia de Chiapa, como en muchos otros lugares colonizados, una carrera por controlar los recursos: sobre todo mano de obra. Para esto las leyes fueron cambiando para proteger, en algunos casos, al indio y para limitar el enriquecimiento desmedido de españoles y criollos; sin embargo, el resultado fue totalmente inverso puesto que poco a poco las leyes coloniales instrumentalizaron el resultado contrario, la transferencia de tierras indias a manos españolas (Ruz, 1992b: 48). Sin embargo, la Corona española en el caso de Chiapa intentó al menos una vez poner freno a esta usurpación de tierras.

Si recordamos el sueldo de los primeros alcaldes mayores de la provincia ascendía a 600 pesos y el de Diego de Alegría, alrededor del año de 1600, era de 1050 pesos. Esto explicaría por qué los alcaldes mayores pretendieron en varias ocasiones recuperar esta función administrativa.

Como exponíamos líneas arriba, en el periodo comprendido entre 1570 y 1616 se va a producir un cambio importante en la manera de contar a los tributarios, incluyendo a viudos de ambos sexos y varones solteros como "medios tributarios"; posteriormente, entre 1616 y 1635, se incluiría a las mujeres viudas y solteras como "tercios tributarios" (Obara-Saeki, 2012: 39) (ver tabla 3).

Los datos que podemos encontrar en la lista anterior corresponden a algún momento entre 1609 y 1610, precisamente la fecha en que la administración de la provincia estaba bajo el resguardo de Diego de Alegría. En la información secreta de los méritos y servicios de este personaje presentado en 1600/1601, uno de los testigos del interrogatorio en información secreta, Pedro de Velasco, afirma que Diego de Alegría realizó padrones de tributarios, ${ }^{24}$ información que será reiterada por otros vecinos en el interrogatorio de 1620: "[Juan Palomino de Vargas, escribano público, dijo que Diego de Alegría] en sus visitas llevaba mucha gente y oficiales para hacer cuentas y padrones."25

Por esto consideramos que la lista de 1611, que ObaraSaeki encontró, fue elaborada por Diego de Alegría en sus sucesivas visitas a los pueblos de la provincia, ya que éste realizaba la cobranza del servicio del tostón personalmente por ser esta provincia "dilatada y de mayor número de indios que hay en el distrito de la Real Audiencia." ${ }^{26}$ Aunque de esto último no podemos estar totalmente seguros. 


\section{Diego de Alegría y el cabildo}

Desde la conquista hasta el nombramiento de corregidores y alcaldes mayores las posesiones coloniales eran reguladas por grupos de conquistadores que tomaban todas las decisiones del gobierno de las "repúblicas de españoles" a través del cabildo. Esta situación cambió en el caso de la provincia de Chiapa en 1578, cuando se nombró al primer alcalde mayor, Juan de Mesa Altamirano, con sueldo de 600 pesos. ${ }^{27}$ A partir de ese momento, toda decisión que quisiera promulgar el cabildo requería de la ratificación de la Corona a través de su delegado, el alcalde mayor. La reacción del cabildo no se hizo esperar y en 1583 solicitó cédula real para que no se nombraran corregidores ni alcaldes mayores, sino sólo alcaldes ordinarios. ${ }^{28}$ No estamos seguros, pero quizá la negativa de estos nombramientos por parte del cabildo se deba a que con ellos perdían cualquier control sobre el cobro de los tributos, puesto que tanto los alcaldes mayores como los corregidores debían realizar esta función.

La Corona española nunca tuvo en mente, al menos durante los siglos XVI y XVII, terminar con la corrupción de sus oficiales, sino que su principal objetivo era que ésta no se saliera de los límites señalados. Recordemos que, como indicábamos al principio del texto, las necesidades financieras de la metrópoli eran muchas y los sueldos de los funcionarios de la administración eran bajos, así que una forma de retribución indirecta era admitir cierto grado de corrupción que permitiera a los funcionarios vivir con el decoro que merecía su cargo. Estos intentos de limitación fueron infructuosos, en gran medida, como señala Bertrand, porque su aplicación hubiera terminado con la cohesión del Imperio español; sólo a partir de finales del siglo XVIII las reformas comenzaron a dejarse sentir en el funcionamiento administrativo, cuando la Corona implementó el control sobre los funcionarios y los costos que esto suponía.

Como indicábamos al inicio del texto, la colusión entre autoridades recaudadoras y oligarquías locales era normal. Tan normal que, en el año 1612, Diego de Alegría, teniente de oficiales reales, "fue electo alcalde ordinario de Ciudad Real de Chiapa con general aclamación."

En el acta de elecciones del cabildo ${ }^{29}$ se especifica que el l de enero de 1612 se juntaron justicias y regimiento en el ayuntamiento de Ciudad Real con la finalidad de elegir alcaldes ordinarios, de la santa hermandad y demás oficiales de República. Los cargos en el cabildo fueron distribuidos como se señala en la tabla 4.

Todos los personajes nombrados en esta elección formaban parte de un grupo muy reducido de personas pertenecientes a la oligarquía local; algunos como descendientes de los primeros conquistadores de la provincia y otros por haber contraído matrimonio con hijas de ellos. El linaje de los Tovilla, por ejemplo, se presenta aquí como una muestra de cómo se fueron tejiendo las estrategias para conservar a los miembros de la familia en puestos que los mantenían dentro de la elite local.

Andrés de la Tovilla fue nombrado procurador de Ciudad Real en 1529 junto con Francisco Ortés de Velasco (Nájera, 1993: 26). El iniciador del linaje en Ciudad Real fue encomendero de Copanaguastla, donde, según algunos indicios, parece que se situaba una mina. Es quizá por este último motivo por el que el primogénito de Andrés, llamado Juan de la Tovilla, contrajo nupcias con Isabel Vázquez Rivadeneira, descendiente de Diego Vázquez Rivadeneira, contador de la Real Caja de Chiapa, como vimos con anterioridad. De este matrimonio nacieron varios hijos, pero de entre ellos nos interesan dos: Juan, su hijo mayor, que se casó con María de Velasco, nieta de Francisco Ortés de Velasco; y Francisca, que se casó con Luis Alfonso de Mazariegos, nieto de Diego de Mazariegos. Los Ortés de Velasco son de gran importancia para el conocimiento de la formación de las oligarquías en Chiapas puesto que crearon el único mayorazgo de origen hispano documentado de la provincia colonial (Nájera, 1993: 12). En las siguientes generaciones los Tovilla emparentaron con: los Solórzano, que a su vez estaban relacionados con los Ortés de Velasco desde la primera generación en Ciudad real por haberse casado con dos hermanas de la familia Torres Medinilla; los Rodríguez del Padrón; y los Barahona y Loaysa, descendientes de Sancho 
de Barahona, que fue presidente de la Audiencia de Guatemala. Como vemos, muchos de los apellidos considerados en esta breve y somera descripción aparecen en las elecciones de cabildo de 1612, tanto en los puestos permanentes como en los transitorios (ver figura 1).

Ahora bien, las preguntas que nos planteamos son cómo y por qué deciden integrar a Diego de Alegría en el cabildo. Según Michel Bertrand, las maneras de integrar a un individuo a las redes sociales-familiares que se estaban tejiendo en la etapa colonial son dos: parentesco y dependencia (Bertrand, 2011: 240). En el primer caso, sabemos que Diego de Alegría nunca contrajo matrimonio, ni en España ni en América. Por este motivo, la única forma que tuvieron de incorporarle en el reducido núcleo local fue a través de la creación de una dependencia mutua: alianzas socio-económicas.

En el primer caso, como indica Michel Bertrand (2011: 303), debemos recordar que los oficiales reales, en este caso su representante Diego de Alegría, recaudaban todos los impuestos sobre el comercio y miraban por el buen funcionamiento de los impuestos arrendados a las elites locales. Según testimonio de Pedro de Herrera en 1620, el teniente de oficiales reales cobró las alcabalas hasta que éstas fueron encabezadas por el cabildo de la ciudad, ${ }^{30}$ y los remates de los productos sujetos a monopolio y los confiscados al comercio ilegal. A estas actividades, en nuestro caso, se sumó el ser el encargado de determinar, de acuerdo a los títulos de propiedad, y medir las tierras baldías y realengas en 1600. ${ }^{31}$ Estas circunstancias hacían que el interés por tener buena relación con el responsable administrativo se acrecentara, sobre todo en el caso de la elite local de Chiapa, donde ésta, debido al aislamiento y abandono de la provincia, quizá era menos influyente y contaba con un número reducido de comerciantes.

Como señala Viqueira, una vez al año la autoridad ocupada de la administración de los tributos, en este caso Diego de Alegría, sacaba a remate en la plaza pública de Ciudad Real los productos que tributaban los indios o el derecho a cobrarlos. Esta situación dio pie al conocido como "fraude de los tributos". Es precisamente en el derecho a cobrar los tributos donde la estafa se hacía más patente puesto que la mayoría de las veces los que adquirían este derecho eran prestanombres del teniente de oficiales reales (Viqueira, 1994: 242-243). En efecto, en los interrogatorios consultados hemos encontrado diversos testimonios que indican que Diego de Alegría prestaba dinero a muchos vecinos de Ciudad Real para que pagaran los tributos reales con la justificación de que éstos no se atrasaran y no fuera en detrimento de la Real Hacienda, ${ }^{32}$ lo que hace sospechar que Diego de Alegría utilizaba a varios vecinos como prestanombres en los remates que se llevaban a cabo en Ciudad Real. Debemos recordar que los tenientes de oficiales reales disponían de amplias sumas de dinero y, además, determinaban quiénes de los proveedores y beneficiarios del estado debían ser retribuidos antes que los demás; de esta forma, el cargo de alcalde ordinario le permitía justificar sus acciones escudado en la vara de la justicia.

En algunas ocasiones, las relaciones creadas entre la elite local y los oficiales reales, o como es nuestro caso sus representantes, eran tan fuertes que entraban en confrontación con los visitadores. Sin embargo, parece que Diego de Alegría pronto supo estrechar sus lazos con sus superiores de Guatemala, ya que fue prestamista en víveres de Manuel Ungría y Girón, ${ }^{33}$ el cual le dejó una deuda de 6872 reales que tuvo que reclamar, entre 1623 y 1625 , a sus herederos en Ávila. ${ }^{34}$

\section{Conclusión}

Después de este acercamiento al desenvolvimiento de la Hacienda Real en la provincia de Chiapa, es claro que muchos son los interrogantes por resolver; por ejemplo, es necesario un estudio más profundo de la Real Caja de Chiapa, vigente por escasos nueve años, que nos proporcionaría información sobre los ramos que existían y los principales actores de su recaudación, así como de las relaciones de éstos con el resto de la población y del cobro de los diferentes ramos de la Hacienda Real hasta la llegada de Diego de Alegría como juez y administrador de la misma. Quizá una ausencia más notoria es un estudio profundo de la legislación y su aplicación en cuanto a la implementación y 
desenvolvimiento de la encomienda en la Capitanía General de Guatemala, y más específicamente en la provincia de Chiapa.

El nombramiento de este personaje, según nuestra opinión, aunó la gestión, la administración y el cobro de los tributos en una sola persona; al fin y al cabo, la caja de la Real Hacienda era el destino final de los dividendos fiscales. Ahora bien, nos falta por investigar cuál era la relación de éste con los diferentes intermediarios, ya fueran alcaldes mayores o corregidores.

Diego de Alegría, como subdelegado de los oficiales reales, se encontraba inserto "en el meollo de una estructura administrativa fundamental para el buen funcionamiento del sistema colonial" (Bertrand, 2011: 303). El teniente de oficiales reales, según nuestra opinión, pudo cambiar el curso de la administración fiscal en Chiapa ejerciendo funciones que sobrepasaban sus obligaciones, como el conteo de la población y la medición de tierras realengas, así como otras que no le estaban permitidas: el cobro de tributos y el préstamo de dinero. Ante esta situación, como hemos visto, los representantes del cabildo, y por ende de la elite local, trataron de congraciarse con él mediante su inserción en el sistema político de Ciudad Real a través de su nombramiento como alcalde ordinario en 1612. Aunque en el documento consultado pareciera que Diego de Alegría se opone al desempeño del cargo de alcalde ordinario aduciendo su ejercicio como delegado de los oficiales reales, e incluso que esto le valiera la amonestación del alcalde mayor, se piensa que finalmente sí lo ejerció, como lo demuestra el hecho de que lo incluya entre sus méritos.

Elnombramiento de este primerjuez y administrador de la Real Hacienda en la provincia de Chiapa por Francisco de Sande, presidente de la Audiencia de Guatemala, permitió a los oficiales reales justificar la presencia de un teniente de oficiales reales en la zona. De esta forma, estos últimos se apropiaban de una facultad que nunca habían tenido, la de nombrar un subdelegado contraviniendo las propias leyes de Indias. De hecho, cuando murió Diego de Alegría en el año 1644 los oficiales reales nombraron a Antonio Gregorio de Urrea como nuevo teniente; sin embargo, el alcalde mayor, Melchor Sardo de Céspedes, interpuso una queja que le permitió cobrar los tributos aunque no administrarlos. A partir de entonces, hasta 1659, los alcaldes mayores fueron los encargados de cobrar y administrar la hacienda en la alcaldía mayor de Chiapa. ${ }^{35}$ Será en 1659 cuando los oficiales reales soliciten a la Corona el poder nombrar tenientes en Chiapa, aunque parece que su solicitud fue denegada puesto que hasta la llegada de Damián de Ochaita, en 1669, esta actividad la continuaron realizando los alcaldes mayores.

Damián de Ochaita era tesorero de la caja real en la Audiencia de Guatemala; sin embargo, el 17 de junio de 1672, recibe una licencia para pasar a España después de concluir sus cuentas en la citada caja. Mientras el trámite de licencia se llevaba a cabo, se recibieron noticias de la mala administración de los alcaldes mayores de Chiapa; por ello, se decide enviar a Damián "a que pusiera aquello en debido corriente según su inteligencia y celo que ha demostrado siempre a lo que es su obligación." ${ }^{36}$ El tesorero pasó en Chiapa un año y medio y en 1674 regresó a Guatemala pretextando una enfermedad, donde continuó desempeñando funciones administrativas. Lo más importante de este episodio es que esta estancia permitió, una vez más, justificar el nombramiento de un teniente de oficiales reales en la alcaldía mayor de Chiapa, situación que hubiera continuado igual de no ser por la intervención de Manuel de Maisterra y Atocha (Parrilla, 2011: 21).

\section{Notas}

${ }^{1}$ Fundición de oro en San Cristóbal de los Llanos de Chiapa, 6 de febrero de 1535, AGI, Guatemala, 393, L.1, ff.113V-114.

${ }^{2}$ Fundición de San Cristóbal de los Llanos de Chiapa, 7 de julio de 1536, AGI, Guatemala, 393, L.1, ff.176V-177.

3 Cartas de Audiencia, 21 de noviembre de 1550, AGI, Guatemala, 9A, R.17, N.72.

${ }^{4}$ Caja de Chiapa. Cuentas de Real Hacienda, 1540/1549, AGI, Contaduría, 995.

${ }^{5}$ Cartas de Audiencia, 21 de noviembre de 1550, AGI, Guatemala, 9A, R.17, N.72, f. 2.

${ }^{6}$ Durante los primeros años de la Colonia se estableció la obligación de que los hombres que pasaran a Indias 
fueran casados y llevaran a sus mujeres al término de un año, bajo ciertas penas, como la venida forzosa de las mujeres. Sin embargo, con la finalidad de que la Corona no tuviera que hacerse cargo de mujeres abandonadas, entre otros objetivos, se otorgaban licencias si se demostraba que las mujeres quedaban bien asistidas, si se otorgaba una fianza o depósito y bajo la promesa de retornar en un plazo no superior a dos años. Para más información (Ots, 1934: 138).

${ }^{7}$ El primer teniente de gobernador nombrado por Pedro de Alvarado para Chiapa fue Francisco Ortés de Velasco, al que había conocido en México como procurador (Lenkersdorf, 2001: 248).

${ }^{8}$ Cartas de Audiencia, 21 de noviembre de 1550, AGI, Guatemala, 9A, R.17, N.72, f. 2.

9 La etapa finalizaría con la tasación del oidor Cristóbal Axcoeta en 1572 (Viqueira, comunicación personal).

${ }^{10}$ A finales del siglo XVIII vuelve a aparecer una Caja Real de Chiapa con el establecimiento de la Intendencia de Ciudad Real. Cada asiento de intendencia era también asiento de una Caja Real.

" Parece que Juan de Mesa Altamirano no contó los pueblos de la alcaldía mayor, aunque si cobró por ello (Viqueira Albán, comunicación personal).

${ }^{12}$ Nombramiento de Juan de Mesa, 10 de noviembre de 1578, AGI, Contratación, 5788, L.1, F.116-118; AGI, Guatemala, 114, N.73; AGI, Contratación, 5788, L.1, f.162 V-163; Nombramiento dePedro Martínez, 29 de septiembre de 1586, AGI, Contratación, 5788, L.1, F.201-202; Nombramiento de Alcalde Mayor, 29 de septiembre de 1586, AGI, Patronato, 293, N.9, R.3; Consulta del Consejo de Indias, 9 de junio de 1596, AGI, México, 1, N.42.

${ }^{13}$ RLI, 1860: Libro VI, título 8, ley XXVIII.

${ }^{14}$ Idolatría de los indios: cofradía Los Doce Apóstoles: Suchiapa, AGI, Patronato, 183, N.l, R.ll.

15 Inventario de bienes: Zapata de Cárdenas, Alonso, AGI, México, 263, N.217.

${ }^{16}$ AGI, Guatemala, 35, R.1, N.12.

${ }^{17}$ Diego de Alegría. 10 de febrero de 1594, Archivo General de Indias, Sevilla (AGI), Pasajeros, L.7, E.3471.

18 Informaciones: Diego de Alegría. 1600 / 1601, AGI, Guatemala, 116, N.l, f. l.
${ }^{19}$ Consulta del Consejo de Indias, 19 de junio de 1599, Archivo General de Indias, Sevilla (AGI), SANTA_FE, 1, N.209.

${ }^{20}$ Personas para la contaduría de Panamá, 30 de julio de 1604 , AGI, Panamá, 1, N.198.

${ }^{21}$ Alegría y de Mandojana, Diego de, 1629, AHN, OMCABALLEROS_SANTIAGO, exp.262.

${ }^{22}$ A partir de 1592, los indios tributarios del Imperio español fueron cargados de una quinta parte del tributo tasado por el servicio al rey, además de los demás tributos que pagaban hasta entonces. Pero en Nueva España y Guatemala, cada tributario cargado de cuatro reales (un tostón) en lugar de aquella quinta parte y por eso se llamarían esta carga el "servicio de tostón." Véase Recopilación de leyes de los reinos de las Indias, 1973 [1681], tomo 2, libro 6, título 5, ley 16 (Obara-Saeki, 2010: 102).

${ }^{23}$ AGI, Guatemala, 35, R.1, N. 12, ff. 40V-41.

${ }^{24}$ Informaciones:Diegode Alegría. 1600/1601, AGI, Guatemala, 116, N.l, f. 1.

${ }^{25}$ Informaciones: Diego de Alegría, 1620, AGI, Guatemala, 122, N.12, f.18.

${ }^{26}$ AGI, Guatemala, 122, N.12, f.4.

${ }^{27}$ Nombramiento de Juan de Mesa, 10 de noviembre de 1578, AGI, Contratación, 5788, L.1, F.116-118; AGI, Guatemala, 114, N.73.

${ }^{28}$ Cartas de cabildos seculares, 1583, AGI, Guatemala, 44B, N.9.

${ }^{29}$ AGI, Guatemala, 122, N.12, f.39-44.

${ }^{30}$ AGI, Guatemala, 122, N.12, f.8-8V.

${ }^{31}$ AGI, Guatemala, 116, N.l, ff.9-11.

${ }^{32}$ AGI, Guatemala, 122, N.12.

${ }^{33}$ Manuel de Ungría y Girón fue oidor de la Audiencia de Guatemala desde 1597 y posteriormente visitador de Chiapa. Él mismo indica que visitó la provincia en 1609 después de 36 años de no haberlo hecho. Ver información en Cartas de Audiencia, 13 de abril de 1609, Sevilla (AGI), Guatemala, 13, R.1, N.3, f.l.

${ }^{34}$ Pleito de Diego de Alegría Mandorana, de Guatemala, Juan de Mercado Lezcano, de Arévalo (Ávila), Inés del Río, de Arévalo (Ávila)..., ARCHV, PL CIVILES, PÉREZ ALONSO (F), Caja 2037,7.

${ }^{35}$ AGI, Guatemala, 35, R.1, N.12.

${ }^{36}$ AGI, Guatemala, 35, R.l, N.12, f. 12. 


\section{Bibliografía}

Artola, Miguel (1982), La hacienda del Antiguo Régimen, vol. 42, Madrid: Alianza Universidad.

Bertrand, Michel (2011), Grandeza y miseria del oficio. Los oficiales de la Real Hacienda de la Nueva España, siglos XVII y XVIII, México: FCE.

Chamberlain, Robert S. (1948), "The Governorship of the Adelantado Francisco de Montejo in Chiapas, 1539-1544", en Contributions to American Anthropology and History, vol. IX, núm. 44-47.

Guerra, François-Xavier (1998), "De la política antigua a la política moderna. La revolución de la soberanía”, en Francois-Xavier Guerra y Annick Lempérière (coord.), Los espacios públicos en Iberoamérica. Ambigüedades y problemas. Siglos XVIII-XIX, México: Centro Francés de Estudios Mexicanos/FCE, pp. 109-139.

Jáuregui, Luis (1999), La Real Hacienda de Nueva España, México: Facultad de Economía-Universidad Nacional Autónoma de México.

Jiménez Pelayo, Águeda (2001), “Tradición o modernidad. Los alcaldes mayores y los subdelegados en la Nueva España”, en Espiral, Estudios sobre Estado y Sociedad, vol. VIII, núm. 21, pp. 133-157.

Lenkersdorf, Gudrum (200la), Génesis histórica de Chiapas (1522-1532). El conflicto entre Portocarrero y Mazariegos, México: UNAM.

Lenkersdorf, Gudrum (2001b), Repúblicas de índios. Pueblos mayas en Chiapas, siglo XVI, México: UNAM.

Nájera Coronado, Martha Ilia (1993), La formación de la oligarquía criolla en Ciudad Real de Chiapa. El caso Ortés de Velasco, México: UNAM.

Obara-Saeki, Tadashi (2010), Ladinización sin mestizaje. Historia demográfica del ârea chiapaneca 1748-1813, Chiapas: CONECULTA.

Obara-Saeki, Tadashi (2012), "Estudio crítico sobre el número de tributarios en Chiapas (1560-1817). Una propuesta metodológica para la historia de la población", en LiminaR. Estudios Sociales y Humanísticos, vol. X, núm. 2, pp. 35-54.

Ots Capdequí, José M. (1934), Instituciones sociales de la América española en el periodo colonial, La Plata, Argentina: Biblioteca de Humanidad.
Parrilla Albuerne, Ana María (2011), "El cobro de tributos en la provincia de Chiapa (1685-1693): poderoso caballero es don Dinero", en Rocío Ortiz Herrera y Carlos Uriel del Carpio Penagos (coords.), Seis ensayos sobre la historia de Centroamérica, Chiapas: UNICACH.

Pietschmann, Horst (1996), Las reformas borbónicas y el sistema de intendencias en Nueva España, México: FCE.

León Pinelo, Antonio de y Juan de Solórzano Pereira (1680), Recopilacion de leyes de los reinos de las Indias, mandadas imprimir y publicar por la Magestad católica del rey don Carlos II. nuestro señor, Madrid. 〈http://www. gabrielbernat.es/espana/leyes/rldi/rldi.html〉 [14 de enero de 2013].

Ruz, Mario Humberto (1992a), Copanaguastla en un espejo. Un pueblo tzeltal en el Virreinato, México: CONACULTA/INI.

Ruz, Mario Humberto (1992b), Savia India, floración ladina: Apuntes para una historia de las fincas comitecas (siglos XVIII y XIX), México: CONACULTA.

Viqueira Albán, Juan Pedro (1994), "Tributo y sociedad en Chiapas (1680-1721)” en Historia Mexicana, vol. XLIV, núm. 2, pp. 237-267.

Viqueira Albán, Juan Pedro (1997), Cronotopología de una región rebelde. La construcción histórica de los espacios sociales en la Alcaldía Mayor de Chiapas (1520-1720), (tesis inédita de doctorado), l'École des Hautes Études en Sciences Sociales (EHESS), París.

Viqueira Albán, Juan Pedro y Mario Humberto Ruz (eds.) (2000), Chiapas. Los rumbos de otra historia, México: CIESAS/UNAM/CEMCA/Universidad de Guadalajara.

Viqueira Albán, Juan Pedro (2002), Encrucijadaschiapanecas, México: Tusquets.

Zavala, Silvio (1992), La encomienda indiana, México: Porrúa.

\section{Archivos}

Archivo General de Indias, Sevilla (AGI).

Archivo de la Real Chancillería de Valladolid, Valladolid (ARCHV).

Archivo Histórico Nacional, Madrid (AHN). 
Tabla 1. Resumen de las cuentas dadas por la Caja Real de Guatemala y Chiapa

\begin{tabular}{|c|c|c|c|c|c|}
\hline Caja Real [1549] & $\begin{array}{l}\text { Oros y plata de } \\
\text { fundiciones }\end{array}$ & Cargo total & Descargo & $\begin{array}{c}\text { Alcance líquido } \\
\text { al tesorero }\end{array}$ & $\begin{array}{c}\text { Depósito de los } \\
\text { casados }\end{array}$ \\
\hline Guatemala & $\begin{array}{l}119311 \text { pesos dos } \\
\text { tomines dos granos } \\
\text { de buen oro }\end{array}$ & $\begin{array}{l}168330 \text { pesos } \\
\text { dos tomines y } \\
\text { tres granos de } \\
\text { oro fino }\end{array}$ & $\begin{array}{l}148152 \text { pesos } \\
\text { dos tomines y } \\
\text { ocho granos de } \\
\text { oro }\end{array}$ & $\begin{array}{l}119477 \text { pesos } \\
\text { siete tomines } \\
\text { siete granos de } \\
\text { oro }\end{array}$ & \\
\hline Chiapa & $\begin{array}{l}10170 \text { pesos diez } \\
\text { granos }\end{array}$ & $\begin{array}{l}10170 \text { pesos } \\
\text { diez granos } \\
\text { (quintos de las } \\
\text { fundiciones de } \\
\text { plata; penas } \\
\text { de cámara y } \\
\text { tributos de los } \\
\text { pueblos) }\end{array}$ & $\begin{array}{l}7378 \text { pesos seis } \\
\text { tomines ocho } \\
\text { gramos de oro }\end{array}$ & $\begin{array}{l}2801 \text { peso dos } \\
\text { tomines } 1.5 \\
\text { grano de buen } \\
\text { oro }\end{array}$ & $\begin{array}{l}1696 \text { pesos } \\
\text { cuatro tomines } \\
\text { seis granos de } \\
\text { oro }\end{array}$ \\
\hline
\end{tabular}

Fuente: Cartas de Audiencia, 21 de noviembre de 1550, AGI, Guatemala, 9A, R.17, N.72, ff. 1-2V.

Tabla 2. Datos sobre los ingresos de los administradores de la Hacienda en Chiapa durante 1689

\begin{tabular}{|c||c||c||}
\hline $\begin{array}{c}\text { Cobranza del servicio del tostón } \\
\text { [En Chiapas se contabilizan más } \\
\text { de } \mathbf{1 6} \mathbf{0 0 0} \text { indios tributarios] }\end{array}$ & $\begin{array}{c}\text { Sueldo otorgado por Francisco de } \\
\text { Sande (1595) }\end{array}$ & $\begin{array}{c}\text { \% por la cobranza y administración } \\
\text { de tributos }\end{array}$ \\
\hline \hline 225 pesos & 150 pesos & $\begin{array}{c}6 \% \text {; se calcula que éstas importarían } \\
600 \text { pesos. }\end{array}$ \\
\hline
\end{tabular}

Fuente: Cartas de Audiencia. 12 de mayo de 1693/16 de marzo de 1697, AGI, Guatemala, 35, R.1, N.12.

Tabla 3. Número de tributarios en el área chiapaneca

\begin{tabular}{|c|c|c|c|c|c|c|}
\hline & Chiapa & Acala & Chiapilla & Ostuta & Pochutla & Suchiapa \\
\hline $\begin{array}{l}\text { Algún momento entre } \\
1609 \text { y } 1610\end{array}$ & 1665 & 365 & 60 & 415 & 155.5 & 200.5 \\
\hline
\end{tabular}

Fuente: Obara-Saeki (2010: 113: 111). Las fechas fueron cambiadas de acuerdo con la información facilitada por Viqueira Albán (comunicación personal). 
Tablas 4. Extracto de información referente a la elección de diversos cargos para el cabildo de Ciudad Real. Miembros del cabildo de 1611

\begin{tabular}{|l|l||}
\hline Alcalde mayor & Gabriel de Loarte y Aguirre \\
\hline \hline Alcaldes ordinarios & Sancho de Herrera, Joan del Coz \\
\hline \hline Alférez & Joan de la Tovilla \\
\hline \hline Alguacil mayor & Miguel Rodríguez del Padrón \\
\hline \hline Regidores & $\begin{array}{l}\text { Pedro Ortés de Velasco, Pedro de Solórzano, Juan de } \\
\text { Avendaño, Luís Morales de Villavicencio }\end{array}$ \\
\hline \hline Depositario general & Gabriel Álvarez de Toledo \\
\hline
\end{tabular}

Tablas 5. Resultado de las elecciones de 1612

\begin{tabular}{|l||l||}
\hline Alcaldes ordinarios & Hernando del Coz, Diego de Alegría \\
\hline \hline Alcaldes de la Santa Hermandad & Francisco Ortés de Velasco, Acacio de Solórzano \\
\hline \hline Fiel ejecutor & Alonso de Aguilar \\
\hline \hline Procurador & Sancho de Herrera \\
\hline
\end{tabular}

Fuente: Informaciones: Diego de Alegría, 1620, AGI, Guatemala, 122, N.12, ff. 39V y 41-41V.

Figura 1. Varias generaciones del linaje de los Tovilla

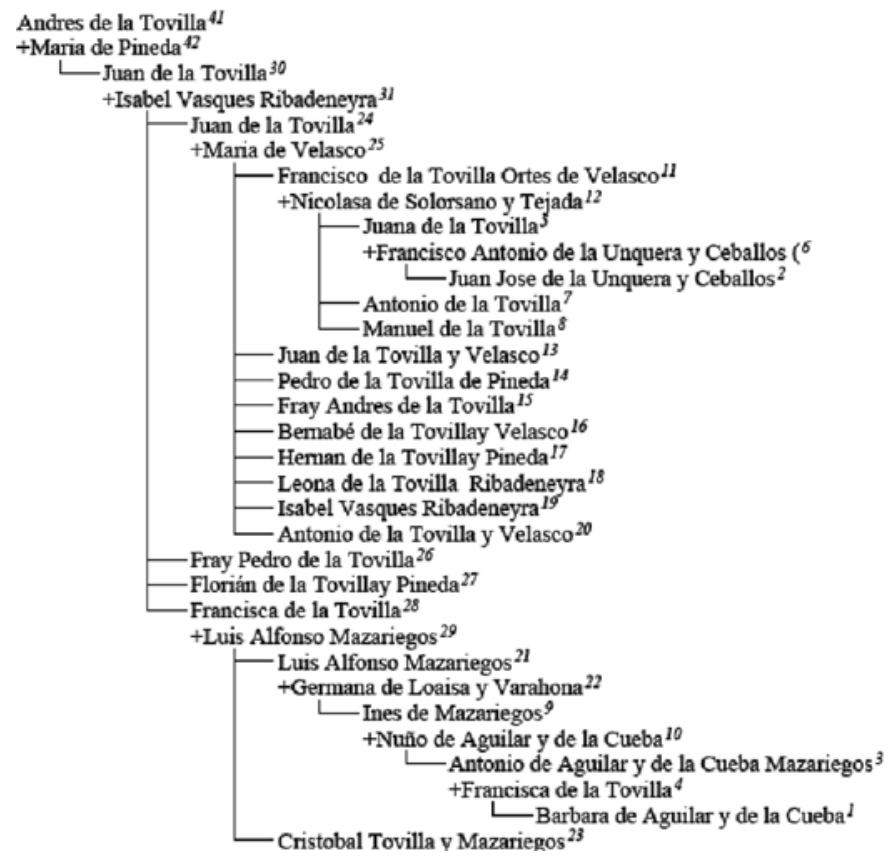

Fuentes: Informaciones: Bachiller Florián de la Tovilla y Pineda, 1606, AGI, Guatemala, 116, N.10; Méritos: Nuño de Aguilar y de la Cueva, probable 17 de marzo de 1631, AGI, Indiferente, 11l, N.104; Méritos: Luis Alfonso de Mazariegos, probable 1609, AGI, Indiferente, 161, N.22; Méritos: Martín Alfonso de la Tovilla, 34 de abril de 1634, AGI, Indiferente, 11l, N.169. 